\title{
Neisseria gonorrhoeae selectively suppresses the development of Th1 and Th2 cells, and enhances Th17 cell responses, through TGF- $\beta$-dependent mechanisms
}

\author{
Y Liu $^{1}$, EA Islam ${ }^{2}$, GA Jarvis ${ }^{3,4}$, SD Gray-Owen ${ }^{2}$ and MW Russell ${ }^{1}$
}

Infection with Neisseria gonorrhoeae does not induce specific immunity or immune memory. Our previous studies in a murine model of vaginal gonococcal infection showed that innate immunity governed by Th17 cells was a critical aspect of the immune response elicited by this pathogen. Herein we show that $N$. gonorrhoeae selectively inhibited Th1 and Th2 cells and enhanced Th17 cell development through the induction of TGF- $\beta$. Whereas Th17 responses depended on gonococcal lipooligosaccharide acting through TLR4, the inhibitory effect of $N$. gonorrhoeae on Th1/Th2 responses involved gonococcal Opa proteins. In vitro Th17 responses to $N$. gonorrhoeae could be diverted to Th1/Th2 by blockade of TGF- $\beta$, but not by blockade of IL-17. The results reveal that $N$. gonorrhoeae suppresses Th1/Th2-mediated adaptive immune response through mechanisms dependent on TGF- $\beta$, and that this effect can be manipulated to promote the development of adaptive immunity.

\section{INTRODUCTION}

Genital tract infection with Neisseria gonorrhoeae typically triggers an intense inflammatory response characterized by an influx of neutrophils, yet the natural infection does not induce a state of effective, specific, protective immunity against re-infection. ${ }^{1,2}$ However, the mechanisms responsible for the lack of protective immunity to N. gonorrhoeae are controversial. It is generally believed that $N$. gonorrhoeae can evade host immune defenses by a combination of strategies including phase-variation and hypervariability of most of its surface antigens, resistance to complement-mediated bacteriolysis, and possibly the production of IgA1 protease. ${ }^{1,3,4}$ Lack of an immune response may also be partially attributable to the absence from the genital tract of organized follicular lymphoepithelial tissues that are considered to be critical for the generation of mucosal immune responses, ${ }^{5}$ as well as to an immunoregulatory and immunosuppressive environment, particularly in the female tract, that permits the growth of the fetus. ${ }^{6}$ However, increasing evidence indicates that $N$. gonorrhoeae can down-regulate specific immune responses in a more direct manner. Transient declines in CD4 + T cell counts and CD8 $+\mathrm{T}$ cell responses in blood have been reported during acute gonococcal cervicitis, which resolved after clearance of the infection. ${ }^{7,8}$ Although specific antibodies associated with $N$. gonorrhoeae infection have been detected in some studies, levels are generally low and short-lived. ${ }^{2,9-11}$ Responses to gonococcal infection of the rectum, which contains lymphoid follicles that serve as an inductive site for mucosal immune responses, are also weak. ${ }^{10}$ In addition, epidemiologic and clinical studies provide strong evidence that gonorrhea predisposes individuals to HIV-1 and chlamydia infection, ${ }^{12,13}$ although the mechanisms responsible for this are not known.

$N$. gonorrhoeae has been shown to suppress the activation and proliferation of human T-helper cells through the interaction of its opacity (Opa) proteins with carcinoembryonic antigen-related cellular adhesion molecule (CEACAM) 1 which is expressed on activated CD $4+\mathrm{T}$ cells. ${ }^{14}$ Opa-CEACAMdependent suppression of $\mathrm{B}$ cell and antibody responses has also been reported. ${ }^{15}$ Collectively these findings suggest that $N$. gonorrhoeae possesses mechanisms to interfere with the

\footnotetext{
${ }^{1}$ Department of Microbiology and Immunology, and Witebsky Center for Microbial Pathogenesis and Immunology, University at Buffalo, Buffalo, New York, USA ${ }^{2}$ Department of Molecular Genetics, University of Toronto, Toronto, Ontario, Canada. ${ }^{3}$ Center for Immunochemistry, Veterans Affairs Medical Center, San Francisco, California, USA. ${ }^{4}$ Department of Laboratory Medicine, University of California, San Francisco, San Francisco, California, USA. Correspondence: Y Liu (yingruli@buffalo.edu) 
development of host adaptive immune responses that might be capable of eliminating it. However, at present, comprehension of the immune response against gonococcal infection and how this can be manipulated to generate protective immunity are limited.

In a mouse model of vaginal gonococcal infection, ${ }^{16}$ it has been shown that $N$. gonorrhoeae induces local inflammation but not acquired immunity or immunological memory. ${ }^{17}$ The infection does not induce a substantial or sustained increase in specific antibodies; moreover, mice can be reinfected with the same strain of N. gonorrhoeae without displaying enhanced resistance, and repeated infection does not boost the antibody response. In these respects, the mouse model reflects well-known features of uncomplicated human gonorrhea.

In the same murine model, we have found that $N$. gonorrhoeae induces Th17-driven innate immune responses. ${ }^{18}$ However, Th1 or Th2 immunity to $N$. gonorrhoeae infection was not evident either in vitro or in vivo. ${ }^{18}$ In the present study we have explored the underlying mechanisms. The results show that $N$. gonorrhoeae can selectively inhibit Th1 and Th2 cell proliferation and cytokine secretion by mouse CD $4+\mathrm{T}$ cells, and concomitantly enhance Th17 activity, by a mechanism dependent upon TGF- $\beta$. Furthermore, whereas gonococcal lipooligosaccharide (LOS) acting through TLR4 is important for eliciting Th17 responses, gonococcal Opa proteins are involved in the suppression of Th1- and Th2-driven immune responses through TGF- $\beta$. In addition, our results demonstrate that blockade of TGF- $\beta$ activity can reverse this trend and elicit Th1 and Th2 responses to N. gonorrhoeae.

\section{RESULTS}

\section{$N$. gonorrhoeae induces TGF- $\beta$ production but not adaptive responses in vitro}

It has been well established that stimulation of human or mouse lymphocytes with different bacteria results in different immune responses characterized by the production of Th lineage-associated cytokines. ${ }^{19-21}$ Our previous data have demonstrated that $N$. gonorrhoeae stimulation elicits a Th17-type response in mouse splenocytes in vitro, ${ }^{18}$ but the underlying mechanisms remain unresolved. In this study, to exclude any potential effect of TGF- $\beta$ present in animal serum on the immune response to $N$. gonorrhoeae, all the following experiments were performed using serum-free culture medium.

When mouse splenic mononuclear cells were cultured under serum-free conditions with $N$. gonorrhoeae, the majority of proliferating cells were B lymphocytes (Figure 1a). T lymphocytes also showed evidence of activation within $12 \mathrm{~h}$ as indicated by up-regulation of CD69 expression (Figure 1b). Stimulation was maximal over the multiplicity of infection (MOI) range 10:1-100:1; larger numbers of gonococci tended to kill the cultures (data not shown). After $3 \mathrm{~d}$, cells stimulated with $N$. gonorrhoeae produced Th17-type cytokines, including IL-17, IL-6, and IL-22, but no IL-12p70, IL-4, or IL-5 (Figure 1c). In addition, TGF- $\beta$ production was observed in the absence of serum additives (Figure 1c). Moderate concentrations of IFN- $\gamma$ were also detected, but flow cytometric analysis revealed that IFN- $\gamma$ was produced predominantly by NK cells rather than CD $4+\mathrm{T}$ cells (Figure 1d). Whereas the main sources of IL- 17 were CD $4+$ and $\gamma \delta$ T cells (Figure 1e), TGF- $\beta$ was produced in multiple immune cell types, including CD19+, $\mathrm{CD} 4+, \mathrm{CD} 8+, \gamma \delta \mathrm{T}, \mathrm{CD} 11 \mathrm{c}+$, and CD11b + cells (Figure 1f). Time course experiments showed that IL-17 production occurred by $3 \mathrm{~d}$ and peaked at $5 \mathrm{~d}$, and that TGF- $\beta$ production paralleled that of IL-17A (Figure 1g). N. gonorrhoeae also slightly elevated the expression of Foxp3 in CD4 + T cells (Figure 1h).

These results showed that most of the IFN- $\gamma$ and some of the IL-17 were produced by innate immune cells in response to $N$. gonorrhoeae, and that TGF- $\beta$ was produced by a variety of cell types. To examine the specific response of CD4+ $\mathrm{T}$ cells to $N$. gonorrhoeae, similar experiments were performed with purified CD4 $+\mathrm{T}$ cells that were stimulated with $N$. gonorrhoeae in the presence of mitomycin C-inactivated APC. A typical Th17 response was observed; at $\mathrm{d} 3$, supernatants from the cultures showed production of IL-17, IL-6, IL-22 and TGF- $\beta$ (Figure 2a). However, the production of Th1- or Th2type cytokines, including IFN- $\gamma$, IL-4, and IL-5, was minimal (Figure 2a). Determination of the expression of nuclear transcription factors using RT-PCR revealed that $N$. gonorrhoeae enhanced the expression of ROR $\gamma \mathrm{t}(P<0.01)$, but not T-bet or GATA3, in CD4 + T cells (Figure 2b). All these data reveal that $N$. gonorrhoeae preferentially primes $\mathrm{Th} 17$ but not Th1 or Th2 cells.

To determine the immune response to $N$. gonorrhoeae in the mouse genital tract, vaginal explants were cultured in vitro with or without the organisms. Supernatants were collected after $3 \mathrm{~d}$ and assayed for secreted cytokines. Consistent with the above findings in mouse lymphocyte cultures, IL-6, TGF- $\beta$, IL-17, and IL-22 were detected in the supernatants of vaginal explants cultured with N. gonorrhoeae, whereas IFN- $\gamma$, IL-4, or IL-5 were not detected at any time point (Figure 2c). Even though vaginal tissues released high levels of TGF- $\beta$ constitutively, this was further increased after stimulation with $N$. gonorrhoeae (Figure 2c). When iliac lymph node cells or vaginal explants were obtained from mice that had been infected in vivo or from sham-infected mice, and then further cultured in vitro with $N$. gonorrhoeae, similar levels of cytokine production were obtained (Figure 2d and e). Previous in vivo findings from the murine model of vaginal gonococcal infection have shown that N. gonorrhoeae does not induce strong Th1, Th2, or antibody responses, ${ }^{22}$ and this result supports the concept that gonococcal infection does not induce immune memory that is amenable to recall in vitro.

\section{N. gonorrhoeae suppresses the development of mouse Th1 and Th2 cells while enhancing Th17 cell development}

The previous experiments showed that $N$. gonorrhoeae elicited a typical Th17 response, including the generation of TGF- $\beta$, in both mouse splenocyte and vaginal explant cultures coupled with a paucity of Th1 and Th2 responses. We therefore hypothesized that $N$. gonorrhoeae selectively suppresses Th1/Th2-mediated immunity. To determine whether N. gonorrhoeae could inhibit 

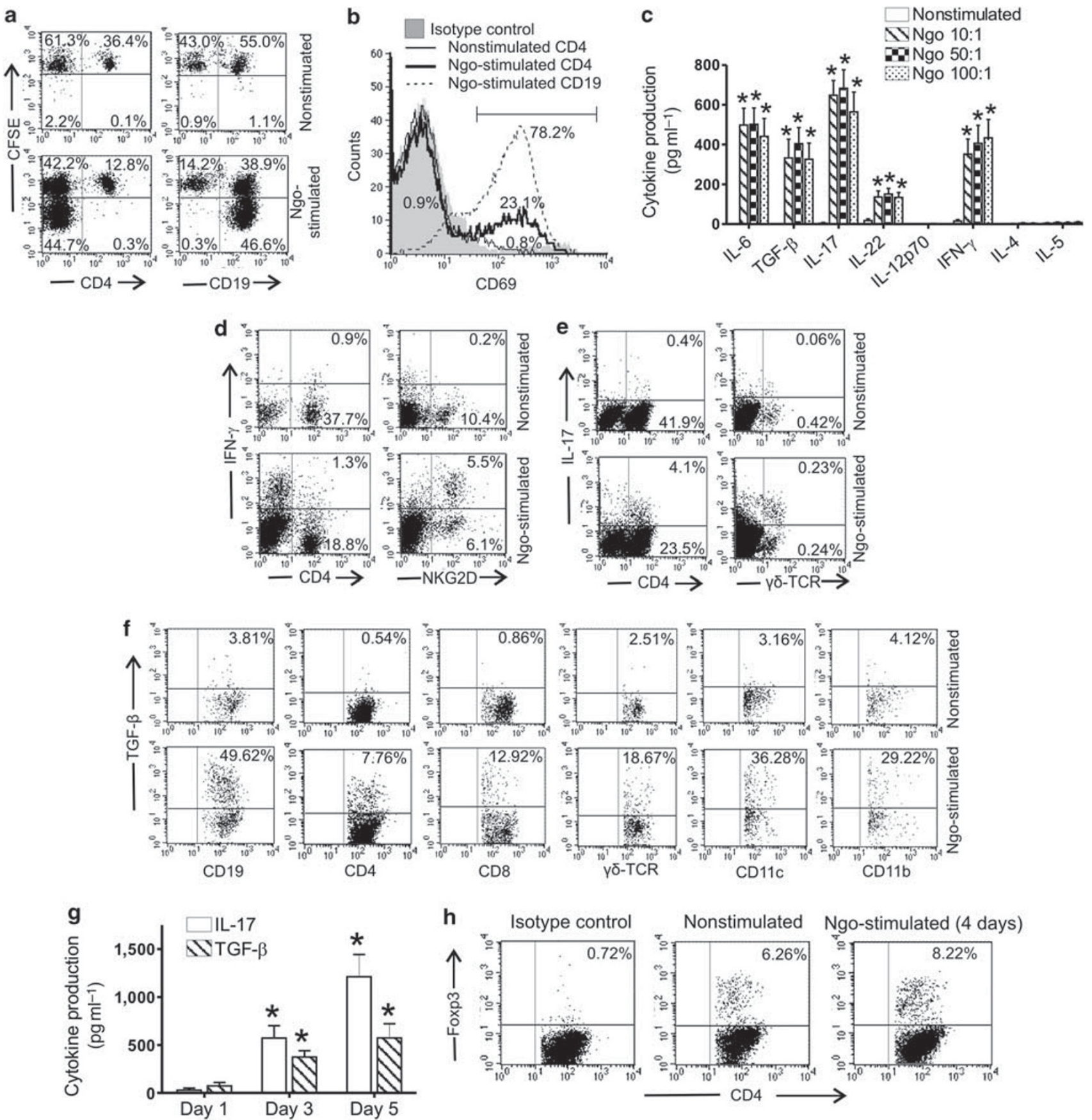

Figure $1 N$.gonorrhoeae (Ngo) activates mouse spleen cells and induces TGF- $\beta$ as a component of the Th17 response. (a) CFSE staining analysis of proliferation of CD4 + T cells and CD19+ B cells in mouse spleenocytes cultured without or with N. gonorrhoeae at MOI 10:1 for $3 \mathrm{~d}$. (b) Activation of mouse spleen T and B cells by $N$. gonorrhoeae (MOI 10:1). CD69 expression in T and B cells after $12 \mathrm{~h}$ of stimulation was analyzed by flow cytometry with gating on live CD4 + or CD19+ cells. (c) Cytokine secretion by mouse spleen cells stimulated with N. gonorrhoeae (MOI 10:1-100:1). After 3d of culture, supernatants were collected and the cytokine levels were measured by ELISA. (d) Intracellular staining for IFN- $\gamma$ in CD4+ vs. NKG2D + cells after stimulation with $N$. gonorrhoeae (MOI 10:1) for $3 \mathrm{~d}$. (e) Intracellular staining for IL-17 in CD4 + vs. $\gamma \delta$ T cells after stimulation with $N$. gonorrhoeae (MOI 10:1) for 3d. (f) Intracellular staining for TGF- $\beta$ in different spleen cell types after stimulation with $N$. gonorrhoeae (MOI 10:1) for 3d. (g) Time course of IL-17 and TGF- $\beta$ production by spleen cells stimulated with $N$. gonorrhoeae (MOI 10:1). (h) N. gonorrhoeae (MOI 10:1) promotes generation of CD4 + Foxp3 + Treg cells in mouse spleen cell culture. The expression of Foxp3 in T cells after $4 \mathrm{~d}$ of stimulation was analyzed by flow cytometry with gating on live CD4+ T cells. All data shown are representative of four independent experiments. ${ }^{\star} P<0.01$ vs. nonstimulated or day 1.

the development of Th1 and Th2 cells, we stimulated purified mouse CD4 + T cells for $4 \mathrm{~d}$ using anti-CD3 antibody under Th1-, Th2-, or Th17-polarizing conditions in the additional presence or absence of N. gonorrhoeae. The results demonstrated that $N$. gonorrhoeae strongly inhibited both cell proliferation and typical cytokine production in Th1- and Th2-polarized cultures, 

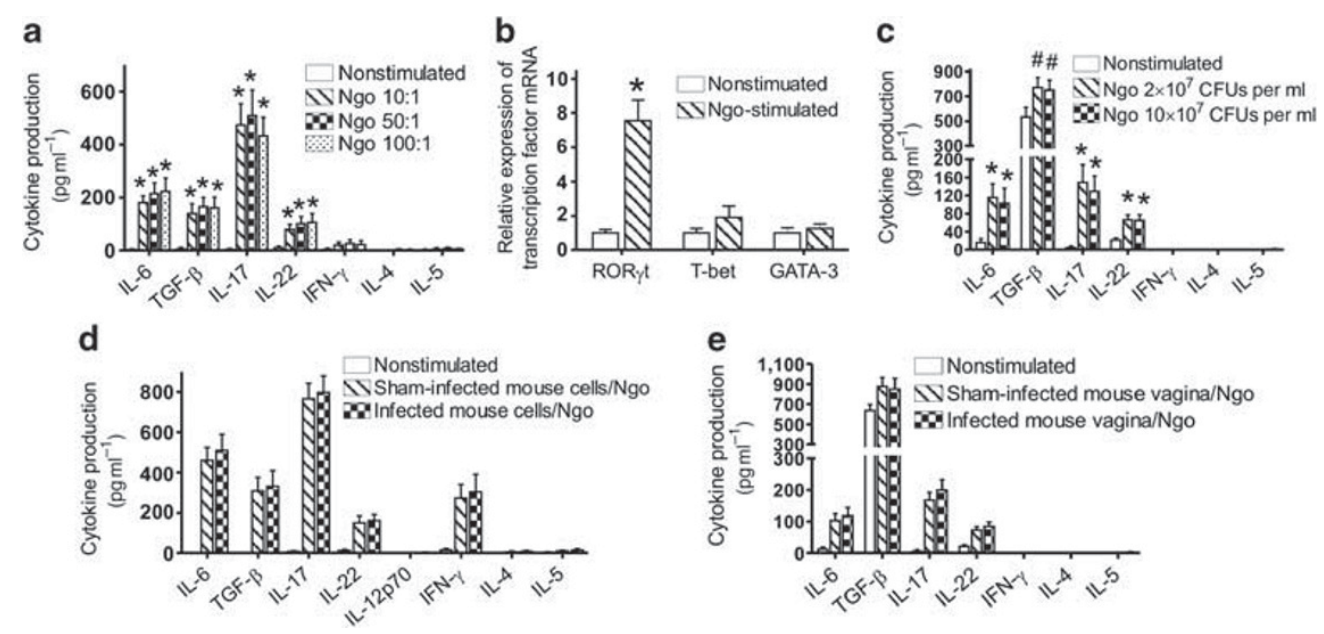

Figure 2 Induction of Th17 cytokines and T cell transcription factors in CD4 + T cells, genital tract explants, and iliac lymph node cells by N. gonorrhoeae. (a) Cytokine secretion by purified CD4 + T cells stimulated with N. gonorrhoeae (MOI 10:1) in the presence of mitomycin C-inactivated APC for $3 \mathrm{~d}$. (b) Expression of Th17 transcription factor ROR $\gamma \mathrm{t}$, Th1 transcription factor T-bet, and Th2 transcription factor GATA3 mRNA in spleen cells stimulated with $N$. gonorrhoeae (MOI 10:1) for $3 \mathrm{~d}$. CD4 + T cells were then purified by FACS, total cellular RNA was isolated, and transcript levels of ROR $\gamma$ t, T-bet and GATA3 were detected by RT-PCR. Transcription factor gene expression levels were normalized with that of $\beta$-actin, which is set at 1.0 for nonstimulated cells. (c) Cytokine secretion by genital tract explants stimulated in vitro with $N$. gonorrhoeae for $3 \mathrm{~d}$. (d) Cytokine secretion by iliac lymph node cells from infected vs. sham-infected mice, stimulated in vitro with $N$. gonorrhoeae (MOI 10:1) for 3d. lliac lymph node cells were collected from mice after $5 \mathrm{~d}$ of infection in vivo. (e) Cytokine secretion by infected vs. sham-infected mouse vaginal explants stimulated in vitro with $2 \times 10^{7} \mathrm{CFUs} / \mathrm{ml} \mathrm{N}$. gonorrhoeae. Vaginal tissues were obtained from the mice after $5 \mathrm{~d}$ of infection. All data shown are representative of four independent experiments. ${ }^{\#} P<0.05 ;{ }^{*} P<0.01$ vs. nonstimulated.

i.e., IFN- $\gamma$ and IL-5, respectively (Figure $3 \mathbf{a}$ and $\mathbf{b}$ ). The effect was dose-dependent and was diminished at MOI 10:1 (data not shown). In contrast, N. gonorrhoeae further increased IL-17 production by Th17-polarized cell cultures without enhancing cell proliferation, possibly because TGF- $\beta$ was already included in these cultures (Figure 3c).

Inhibition of Th1 and Th2 proliferation and activity by treatment with $N$. gonorrhoeae could be due to either functional suppression or elimination of activated cells. We therefore determined the effect of $N$. gonorrhoeae on CD4 + T cell viability to examine whether gonococcal stimulation increased cell death. Polarized Th1, Th2, and Th17 cell cultures were stimulated with $N$. gonorrhoeae for $96 \mathrm{~h}$ and stained with Annexin $\mathrm{V}$ and propidium iodide to detect apoptosis and necrosis. At an MOI of 50:1 that significantly reduced the proliferative response $N$. gonorrhoeae did not increase Th1 cell death (Figure 3d). Similar results were observed for the effects of gonococcal stimulation on Th2 and Th17 differentiated cells (data not shown).

We next investigated whether the down-regulation of Th1 and Th2 responses by $N$. gonorrhoeae could be attributed to the induction of TGF- $\beta$. The addition of TGF- $\beta$-neutralizing antibody significantly reversed $N$. gonorrhoeae-mediated suppression of Th1 and Th2 cell proliferation by $66.5 \pm 10.1 \%$ $(P<0.001)$ and $57.6 \pm 8.9 \%(P<0.01)$, respectively (Figure $3 \mathbf{e})$. Likewise, suppression of IFN- $\gamma$ production in Th1 cells and of IL-5 production in Th2 cells were significantly reversed by TGF- $\beta$-neutralizing antibody by $45.2 \pm 6.1 \%(P<0.01)$ and $43.1 \pm 5.2 \%(P<0.01)$, respectively (Figure $3 \mathbf{f})$. These results show that TGF- $\beta$ contributes to the strong suppressive effect of N. gonorrhoeae on Th1 and Th2 responses.

\section{Suppression of Th1/Th2 responses by $N$. gonorrhoeae is independent of LOS/TLR4 interactions}

Previous studies have demonstrated that gonococcal LOS is a critical virulence factor for $N$. gonorrhoeae and is a major inducer of the proinflammatory cytokine response to the organisms. ${ }^{23-25}$ Our previous findings showed that the generation of Th17 responses by $N$. gonorrhoeae was largely, but not exclusively, dependent on gonococcal LOS interacting with TLR $4 .{ }^{18}$ To determine whether TGF- $\beta$ production and suppression of Th1/Th2 responses were similarly dependent on LOSTLR4 interactions, spleen cells from TLR4-normal C3H/FeJ, TLR4-deficient $\mathrm{C} 3 \mathrm{H} / \mathrm{HeJ}$, or TLR2-knockout mice for comparison were incubated with $N$. gonorrhoeae in serum-free medium. Whereas $N$. gonorrhoeae-induced IL- 6 and IL-17 production were markedly diminished $(P<0.001)$, TGF- $\beta$ production was only slightly albeit significantly reduced in TLR4-deficient cells $(P<0.05$, Figure 4a). In contrast, TLR2-deficient cells were similarly responsive as wild-type cells for all cytokines tested, and neither TLR4- nor TLR2-deficiency resulted in altered IFN- $\gamma$ or IL-4 production (Figure 4a). In addition, although both intact gonococci and purified LOS from the same gonococcal strain elicited IL-6, TGF- $\beta$, and IL-17 responses in wild-type spleen cells, LOS was generally less stimulatory and it induced little or no IFN- $\gamma$ or IL-4 (Figure $4 \mathbf{b}$ ). In contrast, LOS induced more proliferation among B cells (up to $70 \%$ of cells proliferating with LOS at $0.1-5.0 \mu \mathrm{g} \mathrm{ml}^{-1}$ ) than whole gonococci (up to $49 \%$ of cell 

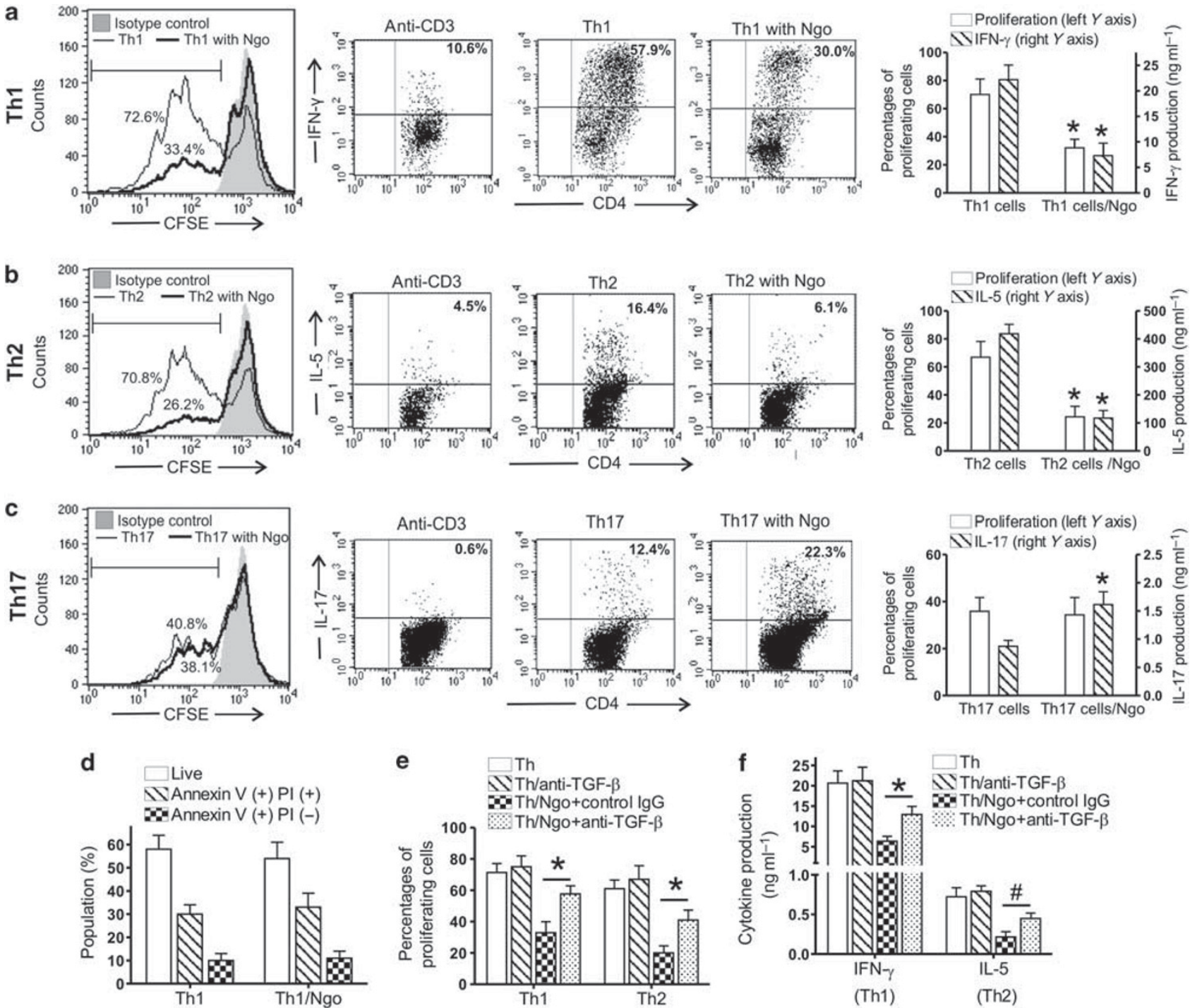

Figure $3 N$.gonorrhoeae suppresses development of Th1 and Th2 cells, but enhances Th17 development, which can be reversed by anti-TGF- $\beta$ treatment. (a) Suppression of Th1 proliferation and IFN- $\gamma$ production by $N$. gonorrhoeae (MOI 50:1) in purified CD4+ T cells stimulated with anti-CD3 under conditions favoring Th1 development. (b) Suppression of Th2 proliferation and IL-5 production by N. gonorrhoeae (MOI 50:1) in CD4+ T cells stimulated with anti-CD3 under conditions favoring Th2 development. (c) N. gonorrhoeae (MOI 50:1) does not affect proliferation of Th17 cells but enhances IL-17 secretion by CD4 + T cells stimulated with anti-CD3 under conditions favoring Th17 development. For (a-c), (left) CFSE staining analysis of proliferation, (center) intracellular IFN- $\gamma$, IL-5, or IL-17 staining, (right) \% of proliferating cells or the production of IFN- $\gamma$, IL-5, or IL-17. (d) Suppression of Th1 development by $N$. gonorrhoeae is not due to increased apoptosis or cell death assessed by Annexin $\mathrm{V}$ and propidium iodide (PI) staining. (e) Suppression of Th1 and Th2 cell proliferation by N. gonorrhoeae is reversed by treatment with anti-TGF- $\beta$ antibody $\left(40 \mu g \mathrm{ml}^{-1}\right)$. (f) Suppression of IFN- $\gamma$ (Th1) and IL-5 (Th2) production by $N$. gonorrhoeae is reversed by treatment with anti-TGF- $\beta$ antibody (40 $\mu$ g ml $\left.{ }^{-1}\right)$. For all above experiments, analysis was performed at $d 4$. Results from one representative out of four independent experiments are shown. ${ }^{\#} P<0.05$; ${ }^{\star} P<0.01$ vs. controls.

proliferating at MOI 10:1-50:1) (Figure 4c). Thus gonococcal LOS acting through TLR4 is important in the generation of Th17 responses, but not in the generation of TGF- $\beta$ and suppression of Th1 or Th2 responses.

Th1, Th2, and to some extent Th17 cells are known to crossregulate the differentiation of each other by means of the cytokines that they produce. We therefore determined whether the induction of a Th17 response through LOS-TLR4 signaling could account for the suppression of Th1/Th2 responses by N. gonorrhoeae. Th1-, Th2-, or Th17-polarized CD4+ T cell cultures were established from TLR4-deficient or wild-type spleens and incubated with $N$. gonorrhoeae, and proliferation and characteristic cytokine production were assessed. Consistent with the above results, deficiency of TLR4 prevented N. gonorrhoeae-induced production of IL-17 by Th17 cells (Figure 4d). However, inhibition of Th1 or Th2 cell proliferation or IFN- $\gamma$ or IL-5 production by $N$. gonorrhoeae was not significantly affected in the absence of TLR4 (Figure 4e and $\mathbf{f}$ ). These data suggest that gonococcal components other than LOS are important for the inhibitory effect of the bacteria on Th1/Th2 immunity. 

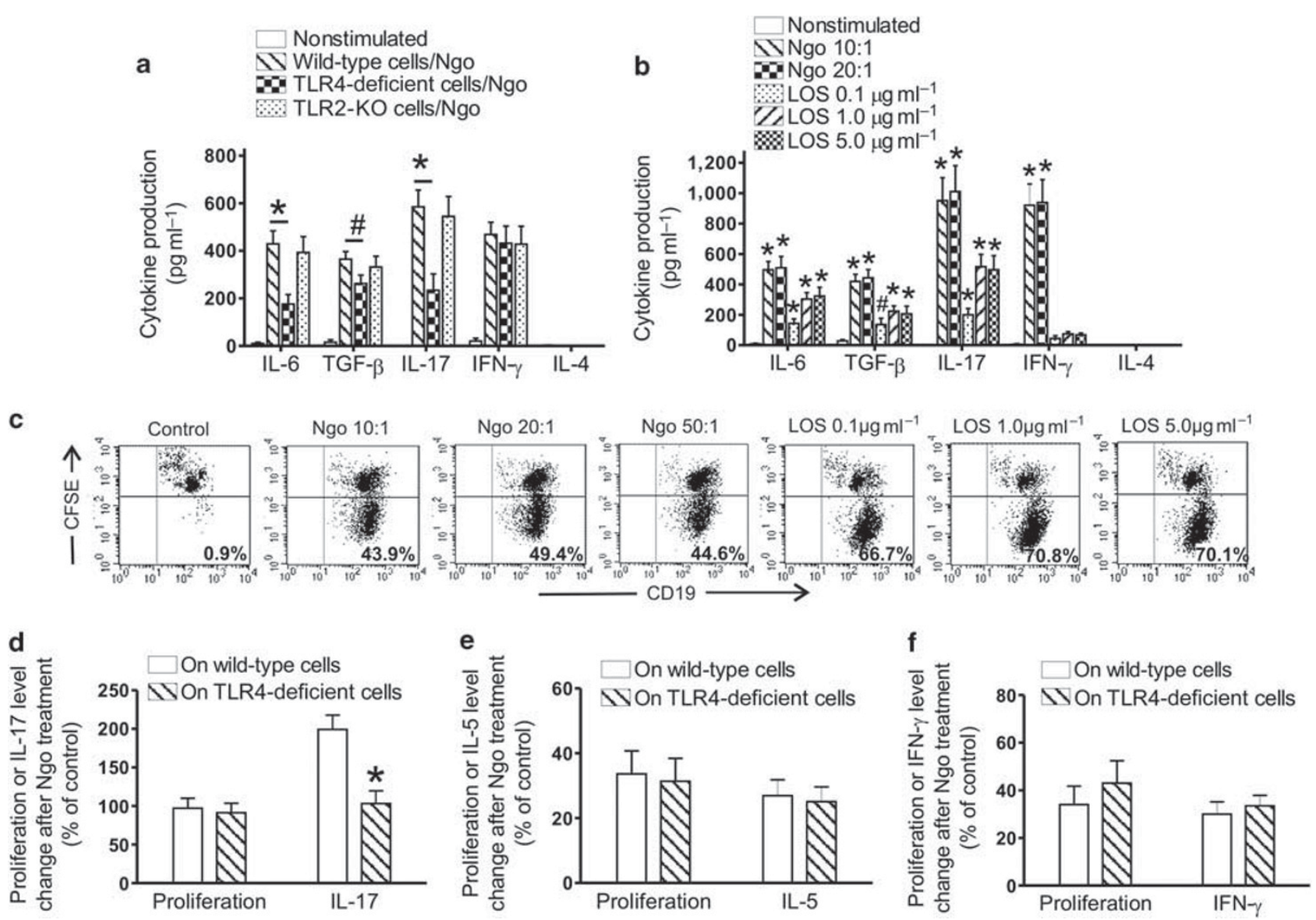

Figure 4 Th17 response to $N$. gonorrhoeae is dependent on TLR4, but deficiency of TLR4 does not compromise the suppression of Th1 and Th2. (a) IL-6, TGF- $\beta$, IL-17, IFN- $\gamma$, IL-4 secretion by wild-type, TLR4-deficient, or TLR2-knockout (KO) mouse spleen cell cultures stimulated with N. gonorrhoeae FA1090 (MOI 10:1) for 3d. (b), IL-6, TGF- $\beta$, IL-17, IFN- $\gamma$, IL-4 secretion by spleen cells cultured with N. gonorrhoeae PID2 or its LOS for $3 \mathrm{~d}$. (c), CFSE staining analysis of proliferation of CD19+ cells in spleen cells cultured with N. gonorrhoeae PID2 or its LOS for $3 \mathrm{~d}$. (d), Enhancement of Th17 by N. gonorrhoeae (MOI 50:1) depends on TLR4. (e), Suppression of Th2 by N. gonorrhoeae (MOI 50:1) is independent of TLR4. (f), Suppression of Th1 by N. gonorrhoeae (MOI 50:1) is not significantly affected by TLR4 deficiency. For experiments shown in panels $\mathbf{d}-\mathbf{f}$, Th17, Th2, and Th1 cells were cultured as described in Methods and analysis was performed at $d 4$; data show the change of cell proliferating rate or Th-type cytokine (IL-17, IL-5, or IFN- $\gamma$ ) production after $N$. gonorrhoeae treatment on both wild-type and TLR4-deficient cells. All data shown are representative of three independent experiments. ${ }^{\#} P<0.05$; ${ }^{\star} P<0.01$ vs. wild-type or nonstimulated cells.

\section{Gonococcal Opa protein is involved in the suppression of Th1/Th2 immune responses}

It has been reported that the interaction of gonococcal Opa proteins with CEACAM1 results in a strong inhibitory effect on human $\mathrm{CD} 4+\mathrm{T}$ cell reactivity. ${ }^{14}$ Opa proteins have also been shown to promote persistence of $N$. gonorrhoeae in the lower genital tract of infected mice. ${ }^{26}$ We therefore evaluated the possible role of Opa in the suppressive activity of $N$. gonorrhoeae against Th1/Th2 immunity, using a mutant of N. gonorrhoeae in which all opa genes have been deleted. ${ }^{27}$ Wild-type N. gonorrhoeae (FA1090) and its Opa-deficient mutant were first tested for Opa protein expression using a panel of monoclonal antibodies in colony dot-blot assays. The results showed that the parent strain expressed Opa $A, B / D / G$, and $\mathrm{E} / \mathrm{K}$, but was negative for $\mathrm{Opa} \mathrm{F} / \mathrm{H}$ and $\mathrm{Opa} \mathrm{C} / \mathrm{J}$, whereas the Opa-deficient mutant did not express any detectable Opa proteins (data not shown). When spleen cells were cultured with wild-type or Opa-deficient N. gonorrhoeae, absence of Opa expression resulted in increased IFN- $\gamma$ production (Figure 5a). No IL-4 or IL-5 production was detected, and there was no significant difference in IL- 6 or IL-17 production between cultures stimulated with Opa-deficient or wild-type $N$. gonorrhoeae (Figure 5a). However, the secretion of TGF- $\beta$ was diminished in the absence of Opa, suggesting that Opa proteins are involved in the induction of TGF- $\beta$ in mouse lymphocytes by N. gonorrhoeae.

Opa-deficiency significantly alleviated the inhibitory effect of $N$. gonorrhoeae on the development of Th1 and Th2 cells (Figure 5b and c), but did not significantly affect Th17 development (Figure 5d). We then investigated whether blockade of the putative receptors for Opa, CEACAM1 or heparan sulfate proteoglycan (HSPG), by blocking antibody AgB10 or heparin, respectively, could abrogate its suppressive effect on mouse Th1 and Th2 reactivity. AgB10 antibody, but not heparin, significantly reversed the inhibitory activity of Opa on the proliferation (Figure 5e) and typical cytokine production 

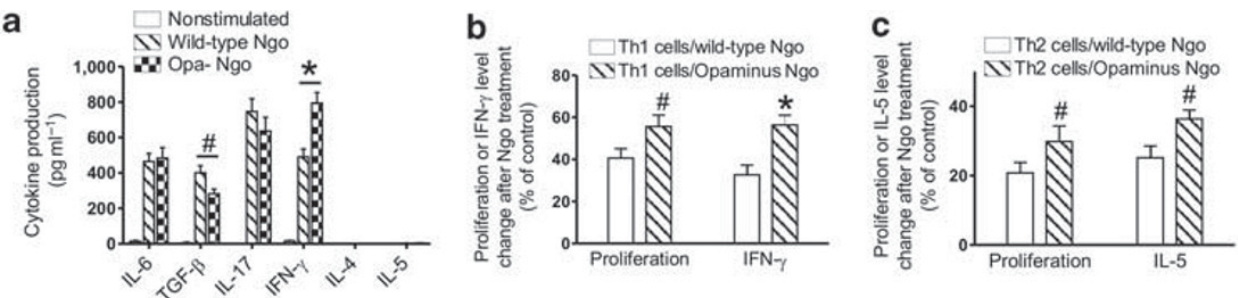

d

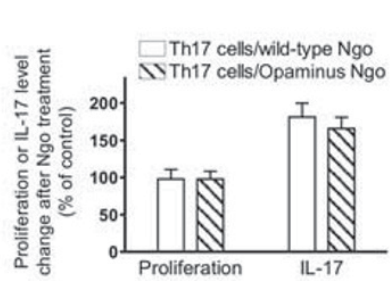

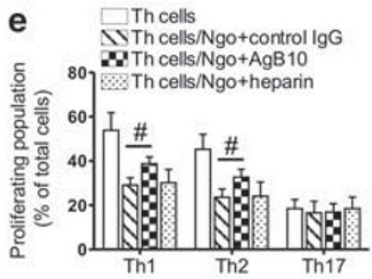
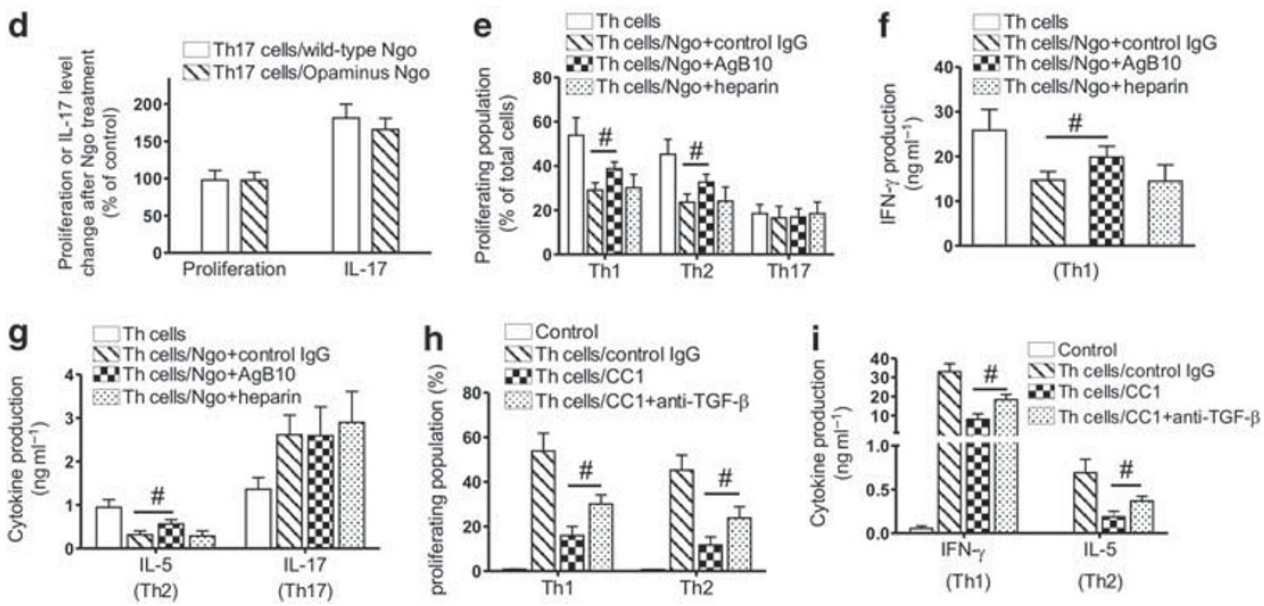

Figure 5 Role of Opa proteins in Th1/Th2/Th17 responses by $N$. gonorrhoeae. (a) IL-6, TGF- $\beta$, IL-17, IFN- $\gamma$, IL-4, IL- 5 secretion by spleen cells stimulated with wild-type vs. Opa- N. gonorrhoeae (MOI 50:1) for 3d. (b) Opa deletion diminishes the suppressive effect of $N$. gonorrhoeae (MOI 50:1) on Th1 cell proliferation and IFN- $\gamma$ production. (c) Opa deletion diminishes the suppressive effect of $N$. gonorrhoeae (MOI 50:1) on Th2 cell proliferation and IL-5 production. (d) Opa deletion does not alter the effect of $N$. gonorrhoeae (MOI 50:1) on Th17 cell proliferation and IL-17 production. (e) Blocking antibody to CEACAM1, AgB10 $\left(5 \mu \mathrm{gml}^{-1}\right)$, partially reverses the inhibition of proliferation of Th1 and Th2 cells due to N. gonorrhoeae (MOI 50:1); heparin $\left(100 \mu \mathrm{g} \mathrm{ml}^{-1}\right)$ has no effect. (f, $\left.\mathbf{g}\right)$ Blocking antibody to CEACAM1, AgB10 $\left(5 \mu \mathrm{g} \mathrm{ml}^{-1}\right)$, partially reverses the inhibition of cytokine secretion by Th1 (IFN- $\gamma$ ) and Th2 cells (IL-5) due to N. gonorrhoeae (MOI 50:1); heparin $\left(100 \mu \mathrm{g} \mathrm{ml}^{-1}\right.$ ) has no effect. (h) Stimulating antibody to CEACAM1, CC1 $\left(1.0 \mu \mathrm{g} \mathrm{ml}^{-1}\right)$, inhibits the proliferation of mouse spleen Th1 and Th2 cells; anti-TGF- $\beta$ antibody $\left(40 \mu \mathrm{g} \mathrm{ml}^{-1}\right)$ partially reverses this. (i) Stimulating antibody to CEACAM1, CC1 $\left(1.0 \mu \mathrm{g} \mathrm{ml}^{-1}\right)$, inhibits IFN- $\gamma$ and IL-5 production in mouse Th1 and Th2 cells respectively; anti-TGF- $\beta$ antibody $\left(40 \mathrm{\mu g} \mathrm{ml}^{-1}\right)$ partially reverses this. For experiments shown in panels $\mathbf{b}-\mathbf{i}$, Th1, Th2, and Th17 cells were cultured as described in Methods and analysis was performed at $\mathrm{d} 4$. All data shown are representative of three independent experiments. ${ }^{\#} P<0.05$; ${ }^{*} P<0.01$ Opa-deletion vs. wild-type N. gonorrhoeae or control treatments.

(Figure $\mathbf{5} \mathbf{f}$ and $\mathbf{g}$ ) of Th1 and Th2 cells. In control cultures without $N$. gonorrhoeae treatment, AgB10 antibody or heparin alone did not affect the development of Th1-, Th2-, or Th17differentiated cells (data not shown). Further studies demonstrated that direct ligation of mouse CEACAM1 with activating antibody $\mathrm{CC} 1$ at $1.0 \mu \mathrm{g} \mathrm{ml}^{-1}$ dramatically reduced Th 1 and Th2 cell proliferation and cytokine production, and that the effect of CC1 antibody was reversed by anti-TGF- $\beta$ antibody treatment (Figure $\mathbf{5 h}$ and $\mathbf{i}$ ). To determine whether $N$. gonorrhoeae was capable of interacting with mouse CEACAM1 expressed on cell surfaces, wild-type FA1090 gonococci were incubated with HeLa cells transfected with human or murine CEACAM1 and stained for human or mouse CEACAM1 and N. gonorrhoeae. Whereas N. gonorrhoeae FA1090 was observed adhering to HeLa cells regardless of CEACAM1 expression, human CEACAM1 was effectively recruited by the gonococci to create regions of intense co-localization at points of bacterial attachment (Supplementary Figure S1 online, lower panels). In contrast there was no association of murine CEACAM1 with gonococci (Supplementary Figure $\mathbf{S} 1$ online, upper panels).

\section{Blockade of TGF- $\beta$ diverts the immune response to $N$. gonorrhoeae from Th17 to Th1/Th2}

Since $N$. gonorrhoeae elicited TGF- $\beta$ production in responding lymphocytes and elevated TGF- $\beta$ production in genital tract tissue, we hypothesized that TGF- $\beta$ has a pivotal role in shaping the immune response to gonococcal infection. We therefore predicted that blockade of TGF- $\beta$ would divert the natural innate immune response to $N$. gonorrhoeae to a Th1/Th2-mediated adaptive response. Blockade of TGF- $\beta$ with a neutralizing antibody increased $N$. gonorrhoeae-induced IFN- $\gamma$ production by mouse lymphocytes (Figure 6a) or by purified CD $4+\mathrm{T}$ cells plus inactivated APC (Figure $\mathbf{6 b}$ ), and triggered the production of IL- 4 and IL-5 (Figure 6a and b). As expected, anti-TGF- $\beta$ antibody also significantly decreased IL-17 production by the lymphocytes in response to N. gonorrhoeae (Figure 6a and b). The results were supported by RT-PCR studies of ROR $\gamma \mathrm{t}$, T-bet, and GATA3 expression in N. gonorrhoeae-stimulated CD4 + T cells treated with anti-TGF- $\beta$ antibody in comparison to control antibody (Figure 6c). Similar effects of TGF- $\beta$ blockade were observed in mouse vaginal explant cultures stimulated with N. gonorrhoeae (Figure 6d). However, the levels of IFN- $\gamma$, IL-4 

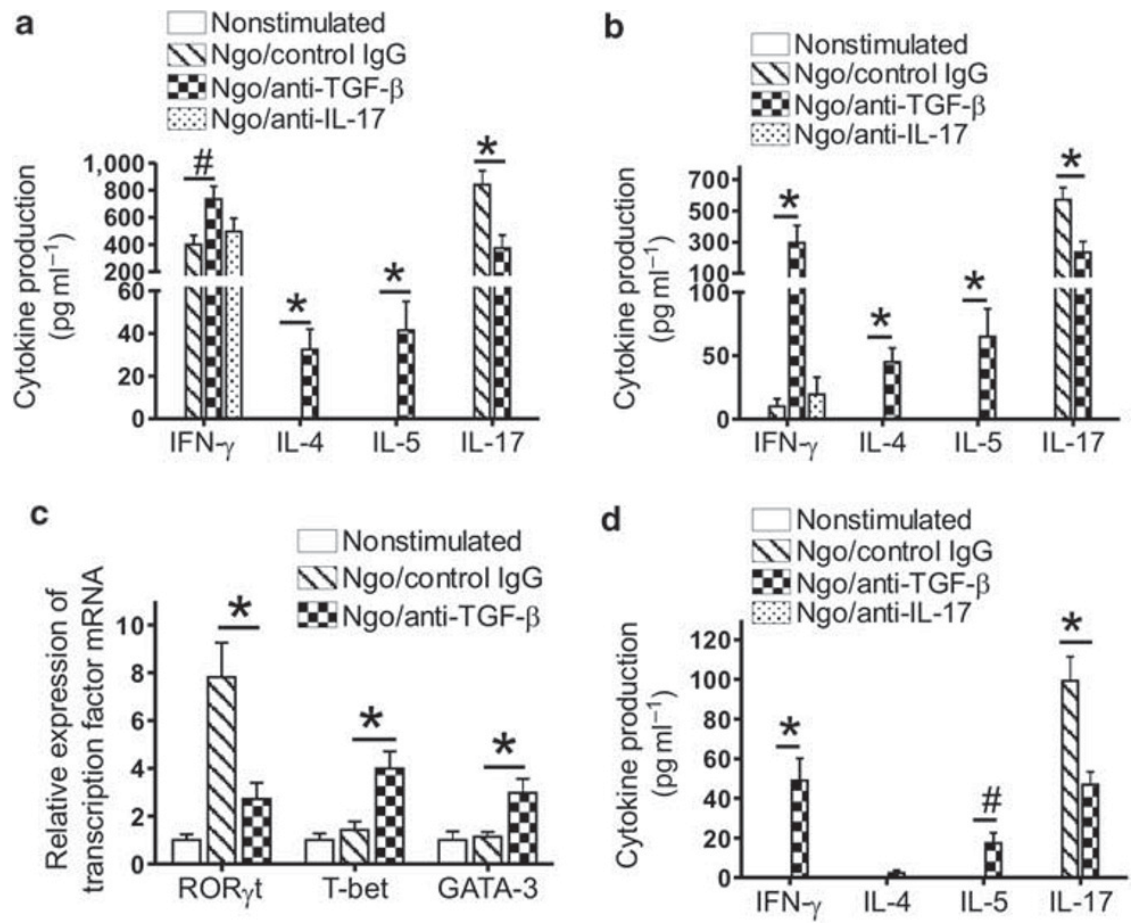

Figure 6 Anti-TGF- $\beta$ antibody treatment suppresses Th17 and promotes Th1/Th2 responses to $N$. gonorrhoeae. (a) Effect of anti-TGF- $\beta$ antibody $\left(40 \mathrm{~g} \mathrm{ml}^{-1}\right)$ vs. anti-IL-17 antibody $\left(20 \mu \mathrm{g} \mathrm{ml}^{-1}\right)$ on cytokine response of spleen cells stimulated with $\mathrm{N}$. gonorrhoeae (MOI 10:1) for $4 \mathrm{~d}$. (b) Effect of anti-TGF- $\beta$ antibody $\left(40 \mu \mathrm{g} \mathrm{ml}^{-1}\right)$ vs. anti-IL-17 antibody $\left(20 \mu \mathrm{g} \mathrm{ml}^{-1}\right)$ on cytokine response of CD4+ T cells stimulated with $N$. gonorrhoeae (MOI 10:1) plus inactivated APC for $4 \mathrm{~d}$. (c) Anti-TGF- $\beta$ antibody $\left(40 \mu \mathrm{g} \mathrm{ml}^{-1}\right)$ inhibits expression of ROR $\gamma \mathrm{t}$ and enhances expression of T-bet and GATA3 in spleen CD4 + T cells stimulated with N. gonorrhoeae (MOI 10:1) for 3 d. (d) Effect of anti-TGF- $\beta$ antibody $\left(40 \mu \mathrm{g} \mathrm{ml}^{-1}\right)$ vs. anti-IL-17 antibody $\left(20 \mu \mathrm{g} \mathrm{ml}{ }^{-1}\right)$ on cytokine response in genital tract explants cultured with $N$. gonorrhoeae $\left(2 \times 10^{7} \mathrm{CFUs} / \mathrm{ml}\right)$ for $4 \mathrm{~d}$. Results from one representative out of four independent experiments are shown. ${ }^{\#} P<0.05 ;{ }^{*} P<0.01$ vs. control treatments.

and IL-5 production that anti-TGF- $\beta$ antibody could support in vaginal cultures were very low possibly because few lymphocytes were present within the tissues. In contrast, antagonism of IL-17 activity by IL-17 neutralizing antibody did not lead to significantly enhanced Th1- or Th2-type cytokine production (Figure 6a, b and d).

\section{DISCUSSION}

Although the lack of a protective adaptive immune response to uncomplicated human gonorrhea has been recognized for a long time, a satisfactory explanation for this situation has not been forthcoming. Symptomatic infection is associated with an intense inflammatory response dominated by the influx of neutrophils, and we have previously shown, in a mouse model of genital tract infection, that this depends on signaling through the principal IL-17 receptor, IL-17RA. ${ }^{18}$ In addition, we proposed that $N$. gonorrhoeae has the capacity to interfere with the development of adaptive immune responses..$^{10}$ We now find in the murine model that, concomitant with the innate response driven by Th17 cells, $N$. gonorrhoeae suppresses the development of adaptive immunity driven by Th1 and Th2 cells through a mechanism dependent upon TGF- $\beta$. Accordingly, we found that $N$. gonorrhoeae induces the generation of TGF- $\beta$ in most mononuclear cell types, including $\mathrm{T}$ and $\mathrm{B}$ cells, and $\mathrm{APC}$, and that it elevates the production of TGF- $\beta$ in the genital tract.
TGF- $\beta$ is well known as a potent regulatory cytokine with diverse effects on immune cell activity, and involved also in the generation of Th17 cells. ${ }^{28}$ In contrast, TGF- $\beta$ inhibits the development of Th1 and Th2 by suppressing the expression and function of the T-bet/Stat 4 and GATA3/NFAT pathways, respectively. ${ }^{29}$ TGF- $\beta$ is also well known to induce Foxp3 expression and the generation of regulatory $\mathrm{T}$ cells. ${ }^{29}$ However, we found that $N$. gonorrhoeae increased the percentage of CD4 + Foxp3 + Treg cells in mouse CD $4+\mathrm{T}$ cells to only a modest extent, possibly because in the additional presence of inflammatory cytokines such as IL- 6 , induced by gonococcal infection, TGF- $\beta$ drives the differentiation of Th17 cells. TGF- $\beta$ directly and indirectly through induced Treg cells suppresses the development of Th1 and Th 2 cells, which are necessary for the generation of specific adaptive immune responses. In contrast, Th17 cells recruit innate defense mechanisms including neutrophils and antibacterial peptides such as defensins, lipocalin-2, and S100 proteins. ${ }^{30}$ Thus the induction of TGF- $\beta$ in numerous immune cell types by $N$. gonorrhoeae, and its enhanced production in genital tract tissues infected with $N$. gonorrhoeae, are probably critical in the selective elicitation of Th17-driven innate inflammatory responses and suppression of Th1/Th2-mediated adaptive immunity by this pathogen.

However, the two pathways of immune response involved different mechanisms of induction by N. gonorrhoeae. Generation 


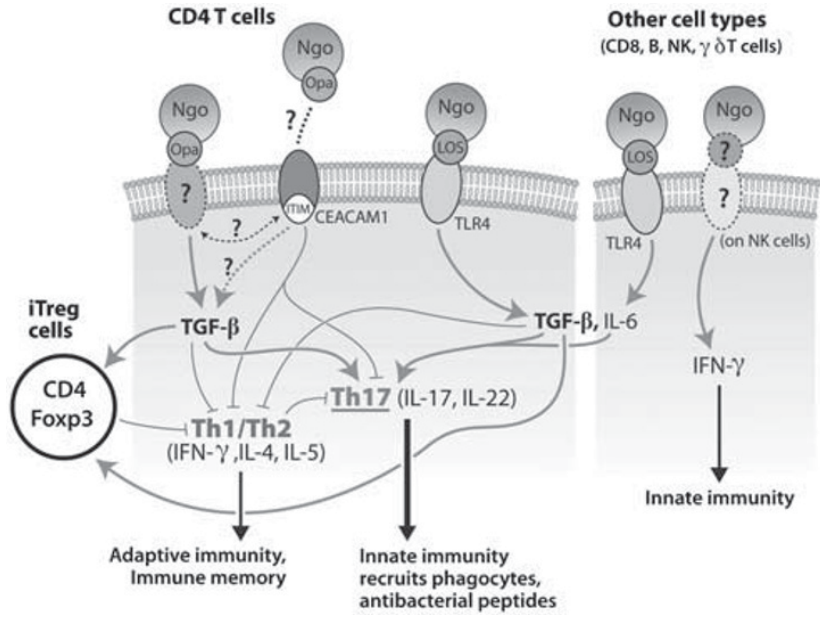

Figure 7 Model for mechanisms of interaction of $N$. gonorrhoeae (Ngo) with cells of the immune system. Gonococcal LOS interacts with CD4+ T cells and other cells through TLR4 to induce the production of IL- 6 and TGF- $\beta$ which drive the development of Th17 cells; IFN- $\gamma$ is also secreted by NK cells. Gonococcal Opa proteins interact with an unknown receptor on mouse cells (or with CEACAM1 on human cells), leading to the increased production of TGF- $\beta$, which directly suppresses Th1 and Th2 cells, and also enhances the development of Treg cells which regulate Th1 and Th2 cells. Blockade of TGF- $\beta$ thus relieves the suppression of Th1 and Th2 cells, which then drive adaptive immune responses, leading to memory and antibody production. (Reprinted from ref. 46; color version available online.)

of Th17 responses depended largely, though not exclusively, on gonococcal LOS interacting with TLR4. This has also been demonstrated for other aspects of the inflammatory cytokine response induced by $N$. gonorrhoeae. ${ }^{23}$ In contrast, TGF- $\beta$ dependent inhibition of Th1 and Th2 responses did not require gonococcal LOS interacting with TLR4, but instead involved gonococcal Opa proteins. In comparison with its wild-type parent, an opa gene-deletion mutant of $N$. gonorrhoeae was defective in inducing TGF- $\beta$ and in suppressing the proliferation and cytokine secretion of Th1 and Th2 cells. A single gonococcus typically expresses 3-4 out of 10-12 Opa proteins at one time, and can switch these off and express other Opa proteins at a rate of approximately $10^{-3}$ to $10^{-4}$ per generation. ${ }^{31}$ Thus wild-type N. gonorrhoeae is Opa-variable, whereas the Opa-negative mutant that we used was completely incapable of expressing any Opa protein. ${ }^{27}$ The FA1090 strain used in our experiments expressed Opa $\mathrm{A}, \mathrm{B} / \mathrm{D} / \mathrm{G}$, and $\mathrm{E} / \mathrm{K}$, all of which bind to human CEACAM1. ${ }^{32}$ The receptor for gonococcal Opa proteins on mouse cells is unknown at this point. The interaction of gonococcal Opa proteins with CEACAM1 on human $\mathrm{CD} 4+\mathrm{T}$ cells results in the inhibition of their activation, ${ }^{14}$ although this was not confirmed in another study. ${ }^{33}$ However, the residues recognized by Opa proteins in human CEACAM molecules are not present in the murine homologs, ${ }^{34,35}$ and we could find no evidence for Opa-dependent interaction of gonococci with murine CEACAM1 expressed on HeLa cells. Nevertheless, blocking antibody to mouse CEACAM1 interfered with the ability of wild-type N. gonorrhoeae to suppress Th1/Th2 activation, whereas blockade of HSPG had no effect. Furthermore, an activating antibody to mouse CEACAM1 strongly induced the inhibition of Th1/Th2 development and this was partially reversed by blockade of TGF- $\beta$, replicating the observed effect of $N$. gonorrhoeae, albeit to a greater extent. The discrepancy may perhaps be explained by postulating that gonococcal Opa proteins interact with an unknown receptor on mouse cells that interacts with intracellular signaling pathways connected to CEACAM1, such that the downstream consequences of either pathway are similar in terms of TGF- $\beta$ production. Antibody-mediated blockade of CEACAM1 might conceivably interfere with the interaction of Opa protein with its unknown receptor, if the two receptors were closely associated on the cell surface, for example if co-located in lipid rafts. ${ }^{36}$ Interestingly, Opa + variants of $N$. gonorrhoeae have been found to be selected during experimental murine vaginal infection, and Opa proteins promote persistence of the bacteria in mouse genital tract. ${ }^{16,26,37}$ However, the evident role of Opa proteins in gonococcal infection of the murine genital tract and disruption of immune responses remains unexplained at this point.

Regardless of the ligand-receptor mechanisms by which $N$. gonorrhoeae interacts with mouse immune cells, it is clear that the functional consequence of these interactions is the concomitant stimulation of Th17-driven innate immunity and inhibition of Th1- and Th2-driven adaptive immune responses (Figure 7). Both are dependent upon TGF- $\beta$ production, through which they enhance each other (Figure 7). N. gonorrhoeae induces Th17 responses including the production of IL-17 by CD $4+$ T cells, as well as other Th17-related cytokines, ${ }^{18}$ and the expression of the nuclear transcription factor, ROR $\gamma$ t which governs Th17 development. In the present studies, N. gonorrhoeae suppressed the development of Th1 and Th2 cells, with inhibition of the production and secretion of the characteristic cytokines including IL-12, IFN- $\gamma$, IL-4, and IL-5, and down-regulation of the master transcription factors, T-bet and GATA3. The importance of TGF- $\beta$ in these responses was clearly demonstrated by experiments in which TGF- $\beta$ was antagonized by means of blocking antibody. AntiTGF- $\beta$ antibody diverted Th17 responses to $N$. gonorrhoeae to Th1/Th2 and reversed the inhibition of proliferation and cytokine production in Th1 and Th2 cells. In contrast, antiIL-17 antibody had minimal effect on Th1 or Th2 immunity. These effects were replicated in genital tract tissue explants cultured with $N$. gonorrhoeae: production of IFN- $\gamma$, IL-4, and IL- 5 were enhanced by anti-TGF- $\beta$ antibody and IL-17 production was diminished. We have further demonstrated the in vivo significance of these effects in the mouse model of vaginal gonococcal infection, in which treatment of mice with anti-TGF- $\beta$ antibody during primary infection accelerated clearance of the infection, facilitated the emergence of Th1 and Th2 responses including the generation of anti-gonococcal antibodies and establishment of immune memory, and enhanced resistance to secondary infection with N. gonorrhoeae. ${ }^{22}$ 
Both human and mouse lymphocytes respond to stimulation by different pathogens with the expression of different patterns of cytokine production, and several species of bacteria are now known to induce Th17 development. ${ }^{19-21,38}$ For example, a Th17 response has also been reported in murine vaginal infection with Chlamydia muridarum. ${ }^{39}$ However, chlamydial infection induced Th1-biased and Th2-biased adaptive immune response as well. ${ }^{39}$ Thus, although $N$. gonorrhoeae is not unique in its ability to induce Th17 immunity, it strongly suppresses Th1/Th2 immunity. On the other hand, Th17-governed innate inflammatory host responses including neutrophil influx are not necessarily the most effective defense against gonococcal infection of the genital tract, at least within humans which are its only natural host. There is growing reason to think that $N$. gonorrhoeae can resist intracellular killing by neutrophils; ${ }^{40,41}$ indeed it utilizes pathogen-directed endocytosis to invade neutrophils. ${ }^{42}$ Multi-drug exporter mechanisms have been shown to facilitate gonococcal resistance to killing by defensins and other antibacterial peptides. ${ }^{43}$ In addition, $N$. gonorrhoeae has developed several mechanisms that inhibit complement activation or resist bacteriolysis. ${ }^{3,44,45}$ Through its long association with humans, $N$. gonorrhoeae has become extremely well adapted to the human immune system, such that it elicits from its host the type of responses that are favorable to its own survival but are not protective of the host. Thus we propose that N. gonorrhoeae illustrates a newly recognized strategy in microbial pathogenesis, in which it is not merely reactive to the responses that the host mounts against it after the pathogen has gained a foothold in the host tissues, but that it proactively directs the manner in which the host responds. ${ }^{46}$ This involves the suppression of adaptive immune responses, including the generation of specific antibodies, that might be effective in eliminating gonococci from host tissues, as well as the induction of immune memory that would provide the basis for protective immunity against repeat infection. Concomitantly, $N$. gonorrhoeae elicits innate immune responses, whether cell-based or molecular, that it can to a large extent survive. This hypothesis is consistent with the known facts of gonococcal pathogenesis and immunity, particularly that the circulating and local mucosal antibody responses to uncomplicated gonorrhea are modest at best and show no evidence of memory, and that gonorrhea can be contracted repeatedly, even by the same serovar of N. gonorrhoeae.

There are three corollaries to this hypothesis. One is that strategies of vaccine development should not seek to mimic the natural infection but instead exploit other approaches to the induction of adaptive anti-gonococcal immune responses, provided that appropriate conserved or cross-reactive antigens can be identified. Secondly, that therapeutic measures might be devised to counteract the ability of $N$. gonorrhoeae to subvert the host immune responses, perhaps by targeting TGF- $\beta$. Thirdly, it is conceivable that the subversion of normal host immune responses by N. gonorrhoeae might contribute to the findings that untreated gonorrhea increases the risk of acquiring or transmitting HIV infection by a factor of up to 5 -fold. ${ }^{12,47}$

\section{METHODS}

Mice. Wild-type BALB/c and C57BL/6 mice were purchased from Harlan Laboratories (Indianapolis, IN). C3H/HeJ (TLR4-deficient), C3H/FeJ (TLR4-normal) and TLR2-deficient (C57BL/6 background) mice were purchased from Jackson Laboratories (Bar Harbor, ME). Mice were maintained under standard conditions in the Laboratory Animal Facility at the University at Buffalo, which is fully accredited by AAALAC. All animal use protocols were approved by the Institutional Animal Care and Use Committee of the University at Buffalo.

Bacteria. N. gonorrhoeae FA1090 48 and an Opa-deletion mutant of FA $1090^{27}$ were kindly provided by Dr Janne Cannon (University of North Carolina at Chapel Hill). N. gonorrhoeae strain PID2 was isolated from a woman with clinically staged pelvic inflammatory disease and has been described previously. ${ }^{25}$ LOS was extracted and purified by a modification of the hot phenol-water method. ${ }^{49}$ Bacteria were cultured on GC agar supplemented with hemoglobin and Isovitalex (BD Diagnostic Systems, Franklin Lakes, NJ) and the resultant growth was checked for colony morphology consistent with Opa protein and pilus expression. Bacteria were harvested from plates and the cell density was determined as detailed previously. ${ }^{18}$

Opa protein typing was performed by colony dot-blot assay ${ }^{16}$ using a panel of monoclonal antibodies specific for the $\mathrm{HV}_{2}$ loops of Opa proteins generously provided by Dr Marcia Hobbs (University of North Carolina at Chapel Hill).

Mouse vaginal infection model. Female BALB/c mice between 7 and 9 wk old were infected with live N. gonorrhoeae FA1090 as previously described by Jerse, ${ }^{16}$ with the modification that water-soluble estradiol was used at day $-2,0$ and $2 .{ }^{18}$ Vaginal mucus was quantitatively cultured on GC agar daily to confirm the infection.

Mouse cell stimulation. Mononuclear cells were isolated from aseptically harvested spleens or iliac lymph nodes using Histopaque 1083 (Sigma-Aldrich, St Louis, MO) density gradient centrifugation. CD4+ T cells were purified through negative selection using a Dynal CD4 cell isolation kit (Invitrogen, Carlsbad, CA). Cells were cultured in 24-well culture plates at a density of $2 \times 10^{6}$ cells $/ \mathrm{ml}$ with either no stimulus, N. gonorrhoeae cells at indicated MOI, or purified LOS of various concentrations. X-VIVO serum-free medium (Lonza, Walkersville, MD) with penicillin/streptomycin was used for all the experiments. For some experiments, mouse TGF- $\beta$ neutralizing $\mathrm{mAb}$ (Bio X Cell, West Lebanon, $\mathrm{NH}$ ) was added.

Th cell differentiation in vitro. Naïve purified spleen CD4 T cells $\left(5 \times 10^{5}\right.$ cells $\left./ \mathrm{ml}\right)$ were cultured in 24 -well tissue culture plates, and stimulated with $1.0 \mu \mathrm{g} \mathrm{ml}^{-1}$ plate-bound anti-CD3 antibody (eBiosciences, San Diego, CA) in complete X-VIVO medium. For Th1 differentiation, cells were stimulated in the presence of $3.5 \mathrm{ng} \mathrm{ml}^{-1} \mathrm{IL}-12$ and $10 \mu \mathrm{g} \mathrm{ml}^{-1}$ anti-IL-4 antibody. For Th2 differentiation, cells were stimulated in the presence of $10 \mathrm{ng} \mathrm{ml}^{-1} \mathrm{IL}-4$ and $10 \mu \mathrm{g} \mathrm{ml}^{-1}$ anti-IFN- $\gamma$ antibody. For Th17 differentiation, cells were stimulated in the presence of $10 \mathrm{ng} \mathrm{ml}^{-1}$ IL-6, $10 \mathrm{ng} \mathrm{ml}^{-1} \mathrm{IL}^{-23}, 5 \mathrm{ng} \mathrm{ml}^{-1}$ TGF- $\beta, 10 \mu \mathrm{g} \mathrm{ml}^{-1}$ anti-IL- 4 antibody and $10 \mu \mathrm{g} \mathrm{ml}^{-1}$ anti-IFN- $\gamma$ antibody. Cytokines and antibodies were purchased from eBiosciences. In each condition, the cells were cultured in the absence or presence of $N$. gonorrhoeae FA1090 for $4 \mathrm{~d}$. Supernatants and cells were then collected for ELISA and flow cytometry analyses. In some experiments, monoclonal activating antibody (CC1; eBioscience) or blocking antibody (AgB10; gift from Dr Nicole Beauchemin, McGill University Cancer Center) to murine CEACAM1, or heparin (SigmaAldrich) was added to investigate the interactions of Opa proteins with putative receptors.

Mouse vaginal explant culture. Mouse genital tracts were dissected out aseptically and washed with HBSS. The tissue was manually separated into about $1 \mathrm{~mm}$ pieces, washed again and weighed. Equal weights were 
added to each well of 24-well cell culture plates. Tissue was incubated in $\mathrm{X}$-VIVO serum-free medium supplemented with penicillin, streptomycin, and fungizone. The explants were cultured with $N$. gonorrhoeae at $2 \times 10^{7}$ or $10 \times 10^{7}$ bacteria/ml, or in medium only as controls. Supernatants were removed at the indicated time points and cytokine production was assayed by ELISA.

Cytokine ELISA. TGF- $\beta$, IL-6, IL-17A, IL-22, IL-12p70, IFN- $\gamma$, IL-4, and IL-5 levels were measured in triplicate using ELISA kits purchased from eBioscience.

Flow cytometry. Cultured cells were washed with staining buffer twice, then incubated with the indicated antibody for $30 \mathrm{~min}$ on ice, washed twice, and analyzed on a FACSCalibur cytometer. For intracellular staining, cells were first fixed with Cytofix/Cytoperm (eBioscience). Antibodies to mouse CD4, CD8, CD19, $\gamma \delta$-TCR, CD11b, CD11c, NKG2D, IL-17A, IFN- $\gamma$, IL-4, IL-5, and Foxp3 conjugated with FITC, PE, or APC were purchased from BD Biosciences or eBioscience. PE-conjugated anti-TGF- $\beta$ antibody was obtained from IQ Products (Groningen, The Netherlands).

Proliferation assays. Cells were labeled with CFSE (Sigma-Aldrich) according to a previous protocol. ${ }^{50} \mathrm{CFSE}-$ labeled cells were then washed twice in PBS, recounted, and stimulated as described in the culture medium. Cultured cells were harvested and then stained with APCconjugated anti-mouse CD4 or anti-mouse CD19 antibody. The data were acquired by gating on the CD $4+$ or CD19+ cell populations in a FACSCalibur cytometer. The sequential loss of CFSE fluorescence was used to measure cell division and proliferation.

Preparation of purified CD4 T cells and real-time RT-PCR. Cultured cells were washed and stained with FITC-conjugated anti-mouse CD4 antibody for $30 \mathrm{~min}$, and labeled CD $4+$ cells were isolated by FACS. Total cellular RNA of purified CD4 + T cells was isolated with RNeasy Mini Kits (Qiagen, Valencia, CA), and was transcribed to cDNA using the iScript cDNA synthesis kit (Bio-Rad, Hercules, CA). Real-time RT-PCR was performed on an iCycler iQ detection system (Bio-Rad) using Sybergreen (Bio-Rad) for real-time monitoring of the PCR. The primers used were as follows: ROR- $\gamma \mathrm{t}, 5^{\prime}$-CCG CTG AGA GGG CTT CAC-3', 5' -TGC AGG AGT AGG CCA CAT TAC-3'; T-bet, 5' -GGA GCG GAC CAA CAG CAT CG-3'; $5^{\prime}$-GCG GGC GGC GGG AAG AAC T-3'; GATA3 5' $^{\prime}$-CCG AAA CCG GAA GAT GTC TA-3'; 5' -AGG GCT CTG CCT CTC TAA CC- $3^{\prime}$; $\beta$-actin, $5^{\prime}$-CCT AAG GCC AAC CGT GAA AAG-3', 5' -GAG GCA TAC AGG GAC AGC ACA-3'. Relative quantification of target genes was analyzed based on the threshold cycle $(\mathrm{Ct})$ determined by Bio-Rad iQ5 optical system software.

Apoptosis assays. Cultured CD4 T cells after indicated treatments were washed twice in PBS. The cell pellets were resuspended in binding buffer containing annexin V-FITC (BD Biosciences) and propidium iodide (BD Biosciences) for $20 \mathrm{~min}$ at room temperature. The samples were analyzed on a FACSCalibur cytometer within $1 \mathrm{~h}$.

Interaction of $\boldsymbol{N}$. gonorrhoeae with CEACAM1-expressing HeLa cells. HeLa cells seeded on glass coverslips were transiently transfected with recombinant expression plasmids encoding human or mouse CEACAM1 as described previously, ${ }^{51}$ and protein expression was confirmed by western immunoblotting analysis of cell extracts. Transfected cells were incubated with wild-type $N$. gonorrhoeae FA 1090 for $1 \mathrm{~h}$ at $37^{\circ} \mathrm{C}$ in culture medium under $5 \% \mathrm{CO}_{2}$, and non-adherent cells removed by washing. After fixation using $3.7 \%$ paraformaldehyde, expressed CEACAM1 was detected by staining with monoclonal antibodies specific for mouse CEACAM1 (generously provided by Dr Kathryn Holmes, Denver, Colorado, USA) or human CEACAM1 (DAKO, Mississauga, Ontario, Canada) followed by BODIPY-FL-conjugated goat anti-mouse Ig secondary antibody. Gonococci were detected using rabbit antiserum ${ }^{52}$ and Texas Red-conjugated goat anti-rabbit Ig. Cells were examined by confocal microscopy.

Statistical analysis. Data are expressed as means \pm s.e.m. Unpaired two-tailed $t$ tests were used to compare the mean values between two groups. Values of $P<0.05$ were considered statistically significant.

SUPPLEMENTARY MATERIAL is linked to the online version of the paper at http://www.nature.com/mi

\section{ACKNOWLEDGMENTS}

We are grateful to the assistance of the Confocal Microscope and Flow Cytometry Facility in the School of Medicine and Biomedical Sciences, University at Buffalo. We thank Dr Marcia M. Hobbs for providing gonococcal Opa-typing antibodies, and Dr Ann E. Jerse for helpful advice and valuable discussions during the course of this work. This work was supported by NIH Grant AI074791 from the National Institute of Allergy and Infectious Diseases, and by a Grant from the John R. Oishei Foundation, Buffalo, NY, to M.W.R. Additional support was provided by the Research Service of the U.S. Department of Veterans Affairs and NIH Grant Al063927 from the National Institute of Allergy and Infectious Diseases to G.A.J., and by an operating Grant from the Canadian Institutes of Health Research (MOP-15499) to S.G.-O.

\section{DISCLOSURE}

The authors declared no conflict of interest.

() 2012 Society for Mucosal Immunology

\section{REFERENCES}

1. Russell, M.W. \& Hook, E.W. 3rd. Gonorrhea. In Vaccines for Biodefense and Emerging and Neglected Diseases (Barrett, A.D.T., Stanberry, L.R., eds) 963-981 Academic Press: London, 2009.

2. Zhu, W. et al. Vaccines for gonorrhea: can we rise to the challenge. Front Microbiol 2, 124 (2011).

3. Ram, S. et al. Binding of C4b-binding protein to porin: a molecular mechanism of serum resistance of Neisseria gonorrhoeae. J Exp Med 193, 281-295 (2001)

4. Smith, H., Parsons, N.J. \& Cole, J.A. Sialylation of neisserial lipopolysaccharide: a major influence on pathogenicity. Microb Pathog 19, 365-377 (1995).

5. Brandtzaeg, P., Kiyono, H., Pabst, R. \& Russell, M.W. Terminology: nomenclature of mucosa-associated lymphoid tissue. Mucosal Immunol 1, 31-37 (2008)

6. Russell, M.W. \& Mestecky, J. Tolerance and protection against infection in the genital tract. Immunol Invest 39, 500-525 (2010).

7. Anzala, A.O. et al. Acute sexually transmitted infections increase human immunodeficiency virus type 1 plasma viremia, increase plasma type 2 cytokines, and decrease CD4 cell counts. J Infect Dis 182, 459-466 (2000).

8. Kaul, R. et al. Gonococcal cervicitis is associated with reduced systemic CD8+ T cell responses in human immunodeficiency virus type 1-infected and exposed, uninfected sex workers. J Infect Dis 185, 1525-1529 (2002).

9. Hedges, S.R., Sibley, D.A., Mayo, M.S., Hook, E.W. 3rd. \& Russell, M.W. Cytokine and antibody responses in women infected with Neisseria gonorrhoeae: effects of concomitant infections. J Infect Dis 178, 742-751 (1998).

10. Hedges, S.R., Mayo, M.S., Mestecky, J., Hook, E.W. 3rd. \& Russell, M.W. Limited local and systemic antibody responses to Neisseria gonorrhoeae during uncomplicated genital infections. Infect Immun 67, 3937-3946 (1999).

11. Price, G.A., Hobbs, M.M. \& Cornelissen, C.N. Immunogenicity of gonococcal transferrin binding proteins during natural infections. Infect Immun 72, 277-283 (2004).

12. Cohen, M.S. Sexually transmitted diseases enhance HIV transmission: no longer a hypothesis. Lancet 351, 5-7 (1998).

13. Hillis, S.D., Nakashima, A., Marchbanks, P.A., Addiss, D.G. \& Davis, J.P. Risk factors for recurrent Chlamydia trachomatis infections in women. Am J Obstet Gynecol 170, 801-806 (1994). 
14. Boulton, I.C. \& Gray-Owen, S.D. Neisserial binding to CEACAM1 arrests the activation and proliferation of CD4+ T lymphocytes. Nat Immuno/ 3, 229-236 (2002).

15. Pantelic, M. et al. Neisseria gonorrhoeae kills carcinoembryonic antigen-related cellular adhesion molecule 1 (CD66a)-expressing human B cells and inhibits antibody production. Infect Immun 73, 4171-4179 (2005).

16. Jerse, A.E. Experimental gonococcal genital tract infection and opacity protein expression in estradiol-treated mice. Infect Immun 67, 5699-5708 (1999).

17. Song, W. et al. Local and humoral immune responses against primary and repeat Neisseria gonorrhoeae genital tract infections of $17 \beta$-estradioltreated mice. Vaccine 26, 5741-5751 (2008).

18. Feinen, B., Jerse, A.E., Gaffen, S.L. \& Russell, M.W. Critical role of Th17 responses in a murine model of Neisseria gonorrhoeae genital infection. Mucosal Immunol 3, 312-321 (2010).

19. Sellge, G. et al. Th17 cells are the dominant T cell subtype primed by Shigella flexneri mediating protective immunity. J Immunol 184, 2076-2085 (2010).

20. Andrews, E., Salgado, P., Folch, H. \& Onate, A. Vaccination with live Escherichia coli expressing Brucella abortus Cu/Zn superoxidedismutase: II. Induction of specific CD8+ cytotoxic lymphocytes and sensitized CD4+ IFN-gamma-producing cell. Microbiol Immuno/ 50, 389-393 (2006).

21. Fedele, G. et al. Lipopolysaccharides from Bordetella pertussis and Bordetella parapertussis differently modulate human dendritic cell functions resulting in divergent prevalence of Th17-polarized responses. $\mathrm{J}$ Immunol 181, 208-216 (2008).

22. Liu, Y. \& Russell, M.W. Diversion of the immune response to Neisseria gonorrhoeae from Th17 to Th1/Th2 by treatment with anti-transforming growth factor $\beta$ antibody generates immunological memory and protective immunity. mBio 2, 00095-11 (2011).

23. Packiam, M., Veit, S.J., Anderson, D.J., Ingalls, R.R. \& Jerse, A.E. Mouse strain-dependent differences in susceptibility to Neisseria gonorrhoeae infection and induction of innate immune responses. Infect Immun 78, 433-440 (2010).

24. John, C.M., Liu, M. \& Jarvis, G.A. Profiles of structural heterogeneity in native lipooligosaccharides of Neisseria and cytokine induction. $J$ Lipid Res 50, 424-438 (2009).

25. Pridmore, A.C. et al. Activation of toll-like receptor 2 (TLR2) and TLR4/ MD2 by Neisseria is independent of capsule and lipooligosaccharide (LOS) sialylation but varies widely among LOS from different strains. Infect Immun 71, 3901-3908 (2003).

26. Cole, J.G., Fulcher, N.B. \& Jerse, A.E. Opacity proteins increase Neisseria gonorrhoeae fitness in the female genital tract due to a factor under ovarian control. Infect Immun 78, 1629-1641 (2010).

27. Jerse, A.E. et al. Multiple gonococcal opacity proteins are expressed during experimental urethral infection in the male. J Exp Med 179, 911-920 (1994).

28. Korn, T., Bettelli, E., Oukka, M. \& Kuchroo, V.K. IL-17 and Th17 cells. Annu Rev Immunol 27, 485-517 (2009).

29. Li, M.O., Wan, Y.Y., Sanjabi, S., Robertson, A.K. \& Flavell, R.A. Transforming growth factor- $\beta$ regulation of immune responses. Annu Rev Immunol 24, 99-146 (2006).

30. Ghilardi, N. \& Ouyang, W. Targeting the development and effector functions of TH17 cells. Semin Immunol 19, 383-393 (2007).

31. Hauck, C.R. \& Meyer, T.F. "Small" talk: Opa proteins as mediators of Neisseria-host-cell communication. Curr Opin Microbiol 6, 43-49 (2003).

32. Fulcher, N.B. The role of Neisseria gonorrhoeae opacity proteins in host cell interactions and pathogenesis. PhD Thesis, University of North Carolina at Chapel Hill (2004).

33. Youssef, A.R. et al. Opa+ and Opa- isolates of Neisseria meningitidis and Neisseria gonorrhoeae induce sustained proliferative responses in human CD4+ T cells. Infect Immun 77, 5170-5180 (2009).
34. Virji, M. et al. Critical determinants of host receptor targeting by Neisseria meningitidis and Neisseria gonorrhoeae: identification of Opa adhesiotopes on the N-domain of CD66 molecules. Mol Microbio/ 34, 538-551 (1999).

35. Villullas, S., Hill, D.J., Sessions, R.B., Rea, J. \& Virji, M. Mutational analysis of human CEACAM1: the potential of receptor polymorphism in increasing host susceptibility to bacterial infection. Cell Microbio/ 9 , 329-346 (2007).

36. Schmitter, T. et al. Opa proteins of pathogenic neisseriae initiate Src kinase-dependent or lipid raft-mediated uptake via distinct human carcinoembryonic antigen-related cell adhesion molecule isoforms. Infect Immun 75, 4116-4126 (2007).

37. Simms, A.N. \& Jerse, A.E. In vivo selection for Neisseria gonorrhoeae opacity protein expression in the absence of human carcinoembryonic antigen cell adhesion molecules. Infect Immun 74, 2965-2974 (2006).

38. Khader, S.A., Gaffen, S.L. \& Kolls, J.K. Th17 cells at the crossroads of innate and adaptive immunity against infectious diseases at the mucosa. Mucosal Immunol 2, 403-411 (2009).

39. Chen, L. et al. Mice deficient in MyD88 Develop a Th2-dominant response and severe pathology in the upper genital tract following Chlamydia muridarum infection. J Immunol 184, 2602-2610 (2010).

40. Criss, A.K., Katz, B.Z. \& Seifert, H.S. Resistance of Neisseria gonorrhoeae to non-oxidative killing by adherent human polymorphonuclear leucocytes. Cell Microbiol 11, 1074-1087 (2009).

41. Criss, A.K. \& Seifert, H.S. Neisseria gonorrhoeae suppresses the oxidative burst of human polymorphonuclear leukocytes. Cell Microbiol 10, 2257-2270 (2008).

42. Jerse, A.E. \& Rest, R.F. Adhesion and invasion by the pathogenic Neisseria. Trends Microbiol 5, 217-221 (1997).

43. Shafer, W.M., Qu, X., Waring, A.J. \& Lehrer, R.I. Modulation of Neisseria gonorrhoeae susceptibility to vertebrate antibacterial peptides due to a member of the resistance/nodulation/division efflux pump family. Proc Natl Acad Sci U S A 95, 1829-1833 (1998).

44. Ram, S. et al. A novel sialic acid binding site on factor $\mathrm{H}$ mediates serum resistance of sialylated Neisseria gonorrhoeae. J Exp Med 187, 743-752 (1998).

45. Lewis, L.A., Burrowes, E., Rice, P.A. \& Ram, S. Interactions of Neisseria with complement. In Neisseria: Molecular Mechanisms of Pathogenesis (Genco, C.A., Wetzler, L., eds) 123-144 Caister Academic Press, Norfolk, UK, 2010.

46. Liu, Y., Feinen, B. \& Russell, M.W. New concepts in immunity to Neisseria gonorrhoeae: innate responses and suppression of adaptive immunity favor the pathogen, not the host. Front Microbio/ 2, 52 (2011).

47. World Health Organization. Global strategy for the prevention and control of sexually transmitted infections: 2006-2015: breaking the chain of transmission (2007).

48. Cohen, M.S. et al. Human experimentation with Neisseria gonorrhoeae: rationale, methods, and implications for the biology of infection and vaccine development. J Infect Dis 169, 532-537 (1994).

49. Liu, M., John, C.M. \& Jarvis, G.A. Phosphoryl moieties of lipid A from Neisseria meningitidis and $N$. gonorrhoeae lipooligosaccharides play an important role in activation of both MyD88- and TRIF-dependent TLR4-MD-2 signaling pathways. J Immunol 185, 6974-6984 (2010).

50. Chen, J.C., Chang, M.L. \& Muench, M.O. A kinetic study of the murine mixed lymphocyte reaction by 5,6-carboxyfluorescein diacetate succinimidyl ester labeling. J Immunol Methods 279, 123-133 (2003).

51. Gray-Owen, S.D., Dehio, C., Haude, A., Grunert, F. \& Meyer, T.F. CD66 carcinoembryonic antigens mediate interactions between Opaexpressing Neisseria gonorrhoeae and human polymorphonuclear phagocytes. EMBO J 16, 3435-3445 (1997).

52. McCaw, S.E., Schneider, J., Liao, E.H., Zimmermann, W. \& Gray-Owen, S.D. Immunoreceptor tyrosine-based activation motif phosphorylation during engulfment of Neisseria gonorrhoeae by the neutrophil-restricted CEACAM3 (CD66d) receptor. Mol Microbiol 49, 623-637 (2003). 